\title{
Differences in COVID-19 Preventive Behavior and Food Insecurity by HIV Status in Nigeria
}

\author{
Morenike Oluwatoyin Folayan ${ }^{1,2}$ - Olanrewaju Ibigbami ${ }^{3} \cdot$ Brandon Brown $^{1,4} \cdot$ Maha El Tantawi ${ }^{1,5}$. \\ Benjamin Uzochukwu ${ }^{1,6}$. Oliver C. Ezechi ${ }^{1,7}$. Nourhan M. Aly ${ }^{1,5}$. Giuliana Florencia Abeldaño ${ }^{1,8}$. \\ Eshrat Ara ${ }^{1,9}$. Martin Amogre Ayanore ${ }^{1,10}$. Oluwagbemiga O. Ayoola ${ }^{1,11}$. Bamidele Emmanuel Osamika ${ }^{1,12}$. \\ Passent Ellakany ${ }^{1,13}$. Balgis Gaffar ${ }^{1,14} \cdot$ Ifeoma Idigbe $^{1,7} \cdot$ Anthonia Omotola Ishabiyi ${ }^{1,15} \cdot$ Mohammed Jafer $^{1,16}$. \\ Abeedha Tu-Allah Khan ${ }^{1,17}$. Zumama Khalid ${ }^{1,18}$. Folake Barakat Lawal ${ }^{1,19}$. Joanne Lusher ${ }^{1,20}$. \\ Ntombifuthi P. Nzimande ${ }^{1,21}$. Bamidele Olubukola Popoola ${ }^{1,22}$. Mir Faeq Ali Quadri ${ }^{1,23}$. Maher Rashwan ${ }^{1,24,25}$. \\ Mark Roque ${ }^{1,26}$. Anas Shamala ${ }^{1,27}$. Ala'a B. Al-Tammemi ${ }^{1,28}$. Muhammad Abrar Yousaf ${ }^{1,29}$. \\ Roberto Ariel Abeldaño Zuñiga ${ }^{1,30}$ • Joseph Chukwudi Okeibunor ${ }^{1,31} \cdot$ Annie Lu Nguyen ${ }^{1,32}$
}

Accepted: 7 August 2021 / Published online: 13 August 2021

(c) The Author(s), under exclusive licence to Springer Science+Business Media, LLC, part of Springer Nature 2021

\begin{abstract}
The aim of the study was to assess if there were significant differences in the adoption of COVID-19 risk preventive behaviors and experience of food insecurity by people living with and without HIV in Nigeria. This was a cross-sectional study that recruited a convenience sample of 4471 (20.5\% HIV positive) adults in Nigeria. Binary logistic regression analysis was conducted to test the associations between the explanatory variable (HIV positive and non-positive status) and the outcome variables-COVID-19 related behavior changes (physical distancing, isolation/quarantine, working remotely) and food insecurity (hungry but did not eat, cut the size of meals/skip meals) controlling for age, sex at birth, COVID-19 status, and medical status of respondents. Significantly fewer people living with HIV (PLWH) reported a positive COVID-19 test result; and had lower odds of practicing COVID-19 risk preventive behaviors. In comparison with those living without HIV, PLWH had higher odds of cutting meal sizes as a food security measure (AOR: 3.18; 95\% CI 2.60-3.88) and lower odds of being hungry and not eating (AOR: $0.24 ; 95 \%$ CI $0.20-0.30$ ). In conclusion, associations between HIV status, COVID-19 preventive behaviors and food security are highly complex and warrant further in-depth to unravel the incongruities identified.
\end{abstract}

Keywords COVID-19 $\cdot$ Food security $\cdot$ HIV $\cdot$ Pandemic $\cdot$ Health behavior

\section{Introduction}

Coronavirus disease-2019 (COVID-19) is an infectious disease caused by a newly discovered coronavirus called severe acute respiratory syndrome coronavirus-2 (SARS-CoV-2). The severity of COVID-19 infection, including risk of death increases with multiple co-morbidities [1]. People with asthma, chronic lung disease, diabetes, serious cardiovascular conditions, chronic kidney disease, obesity, chronic liver disease, and those who are immunocompromised have a higher risk of the disease and are therefore asked to take extra-cautionary measures to avoid contracting the

Morenike Oluwatoyin Folayan

toyinukpong@yahoo.co.uk

Extended author information available on the last page of the article
SARS-CoV-2 infection [2, 3]. Likewise, there were initial concerns that people living with HIV (PLWH) might be at higher risk for COVID-19 outcomes due to the immunosuppressive effect of HIV, resulting in increased susceptibility to opportunistic infections [4]. Prior evidence suggest that HIV is less of a risk factor for severe COVID-19 compared to other health conditions, such as high blood pressure, heart diseases, lung diseases, cancers, overweight/obesity, diabetes, or being over a certain age [5-8]. However, PLWH with low CD4 cell count, advanced disease, high viral load, and those not on antiretroviral treatment may have different health risks [4]. As PLWH are living longer with antiretroviral therapy, many will also have developed the chronic conditions that are associated with severe COVID-19 [9]. Thus, when PLWH contract COVID-19, they are then more likely to have the severe form of the disease and may die at 
a younger age when compared to people not living with HIV [4]. More recent evidence suggests that PLWH are not only at a higher risk for mortality from COVID-19 but are also at a higher risk of contracting SARS-CoV-2 infection than people not living with HIV [10].

In view of PLWH increased risk for COVID-19, it is possible that PLWH may adhere to COVID-19 preventive behaviors. Such COVID-19 preventive behaviors and measures include mask-wearing, frequent hand washing and sanitization, physical distancing, as well as self-quarantine when the need arises. However, lockdown, isolation and quarantine restrict movements making it difficult for PLWH to reach medical clinics for routine care and medications [ 4 , 11-13]. These restrictions can cause disruptions in the continuity of HIV care and cause emotional distress for those who are unable to obtain medical care. Emotional distress can also result from other multiple factors such as food insecurity because of drastic reduction in income and the increasing poverty rates in many countries resulting from the COVID-19 pandemic [14].

Food insecurity, defined as limited or uncertain access to sufficient, nutritious food for an active, healthy life [15], has increased in many countries because of the pandemic [16, 17]. This has resulted in skipping meals or starvation among the affected people because of challenges with accessing basic food needs. The experience of food insecurity is stressful and associated with poorer mental health in the short and long term [18, 19]; and there are indications that this is worse for PLWH [20]. Food insecurity may provoke a stress response that induces mental health conditions like depression. Such stress stimulus may result from having to acquire foods in socially unacceptable ways thereby inducing feelings of alienation, guilt, powerlessness and shame that are associated with depression [20-23]. PLWH are more prone to depression than people not living with HIV [24]. Depression is also associated with health-risk behaviors [25] that increases the risk for non-adherence to COVID-19 precautionary measures.

Food insecurity is the predominant form of uncertainty experienced in daily living in many countries in sub-Saharan Africa [26]. In Nigeria, 58\% of households experienced severe food insecurity during the pandemic [27]. This prevalence of household food insecurity increases to about $71.7 \%$ for PLWH in Nigeria [28]. Nigeria also had the second highest burden of HIV in the world [29] with a HIV prevalence of 1.4\% [30], the highest tuberculosis burden in Africa [31] and a high prevalence of HIV and tuberculosis co-morbidity [32]. PLWH are also at high risk for obesity, diabetes mellitus and cardiovascular diseases [33]. The high prevalence of infectious and non-communicable diseases in PLWH raises concern about their increased risk to COVID-19 related mortality [34] as observed in South Africa [35], a country with a similar HIV, tuberculosis and food insecurity profile like Nigeria. The COVID-19 pandemic increased the risk for food insecurity in Nigeria [36] and hence, a concern for PLWH who often have worse food security problems than the general population [37].

The study assessed if there were significant differences in (1) the adoption of COVID-19 preventive behaviors; and (2) experience of food insecurity among people living with and without HIV in Nigeria. We draw on the stress process model $[38,39]$ that identified the eventful experiences and life strains that produces stress and psychological distress (especially depression [40]) that increases the risk for food insecurity. We hypothesized that PLWH are more likely to adopt COVID-19 preventive behaviors and more likely to experience food security challenges when compared to people not living with HIV.

\section{Methods}

Ethical approval of the current study was obtained from the Human Research Ethics Committee at the Institute of Public Health of the Obafemi Awolowo University Ile-Ife, Nigeria (HREC No: IPHOAU/12/1557).

\section{Study Design, Study Setting and Study Participants}

This study was part of a larger cross-sectional study that used an online survey (Survey Monkey ${ }^{\circledR}$ ) to collect multicountry data to determine the impact of COVID-19 on the mental health and wellness of adults from July to December 2020 [41]. The survey collected data from a convenience sample of adults aged 18 years and above who provided consent for study participation. There were no exclusion criteria for study participation. Data of participants resident in Nigeria who participated in the online survey, were extracted for this study.

\section{Recruitment of Study Participants}

Study participants were recruited through respondent driven sampling. Initial participants reached by the 45 data collectors, were asked to share the survey link with their contacts within their countries to facilitate recruitment. The survey link was also posted on social media groups (Facebook, Twitter, and Instagram) and network email lists and WhatsApp groups.

\section{Data Collection Tool}

Data was collected using a questionnaire that was initially developed for a study that targeted a specific population in the United States and was consequently adapted and validated for global use [42]. The survey questionnaire was 
preceded by a brief introduction explaining the purpose of the study, assuring participants of their voluntary participation, and confidentiality of their data. Study participants provided consent before participating in the online survey. The questionnaire was anonymous, closed-ended and was administered in English and took an average of $11 \mathrm{~min}$ to complete. Each participant could only complete a single questionnaire through IP address restrictions, though they could edit their answers freely until they choose to submit. Study participants were asked to provide details about their sociodemographic profile, medical health profile, COVID19 status, HIV status, feeling of being depressed, behavior change during the pandemic, and COVID-19 impact on their food security.

\section{Control Variables}

\section{Sociodemographic Profile}

The section on sociodemographic profile collected data on age, sex at birth, highest level of education attained (none, primary, secondary, college/university) and employment status.

\section{Medical Health Profile}

The section on medical health profile required respondents to tick one or more of 23 medical health conditions adapted from the study by Marg et al. [43] and classified based on the risk definition by the Centers for Disease and Prevention [44]. There was also an option to mention other health conditions not in the list. The list was constructed to identify respondents whose medical conditions put them at high (pneumonia, diabetes, cancer, heart condition), moderate (hepatitis, hypertension, neurological problems, neuropathy, respiratory problems, stroke, depression) or low (herpes, shingles and other sexually transmitted infections, dermatologic problems, migraines, arthritis, broken bones, hearing loss and vision loss) risk for severe COVID-19.

\section{COVID-19 Status}

Respondents were asked if they had tested positive for COVID-19, had COVID-19 symptoms but did not test, had a close friend who tested positive for COVID-19 and/or knew someone who died from COVID-19.

\section{Depression}

Respondents were asked to indicate which of the eight feelings they were experiencing during COVID-19. They were required to tick a checkbox against any of the feelings they had experienced during the pandemic. One of the eight feelings was depression. The questions were part of the Pandemic Stress Index administered to study participants that assessed the psychosocial impact of COVID-19 [45].

\section{Outcome Variables}

\section{Behavior Changes}

The questionnaire assessed behavior changes to public health messaging (wearing of face masks, frequent washing or sanitizing of the hand, physical distancing, been in isolation or quarantined) and the workplace modification (working remotely). Respondents were asked which of the listed behaviors they had adopted during the pandemic; and were required to check the box against any of the listed behaviors. A check indicated they had adopted that behavior during the pandemic. They questions were included as a component of the Pandemic Stress Index [45].

\section{Impact of Pandemic on Food Security}

The second assessment was the impact of the pandemic on access to food and meals with responses as either a 'yes' or 'no' to the following questions: Were you ever hungry but did not eat because there wasn't enough money for food? Did you ever cut the size of your meals or skip meals because there wasn't enough money for food? The questions were adapted from the US Department of Agriculture Household Food Security Survey [46].

\section{Explanatory Variable}

\section{HIV Status}

A question was also asked about HIV status. Respondents were required to identify if their HIV status was positive, negative, unknown or if they were unwilling to declare.

\section{Data Analyses}

Raw data were downloaded, cleaned, and imported to SPSS ${ }^{\circledR}$ Version 23.0 (IBM Corp. Released 2015. IBM SPSS Statistics for Windows, Version 23.0. Armonk, NY: IBM Corp) for analyses. As a best-practice procedure, we checked to identify and remove any survey responses completed below seven minutes - the lower limit of the time range to answer the questionnaire during the pilot phase $(n=77)$; and those with incomplete data with respect to responses on HIV status $(n=220)[47,48]$.

Descriptive analysis of all study variables was conducted. PLWH and participants not living with HIV were compared regarding background variables, COVID-19 status food insecurity and adoption of precautionary measures using $t$ 
test and chi square test. Logistic regression models were constructed for five dependent variables (the adoption of three precautionary measures and two food insecurity indicators). The COVID-19 precautionary measure variables used to construct the logistic regression were three of the five variables those that showed statistically significant associations with HIV status. The confounders for the study were sociodemographic profile, COVID-19 status, medical status, and depression. HIV status was the explanatory variable. Adjusted odds ratios (AOR) for the binary logistic regression models and $95 \%$ confidence intervals (CIs) were calculated. The Omnibus test of model coefficients was used to determine the significant difference between the Log-likelihoods (specifically the -2LLs) of the baseline model and the new model inclusive of the explanatory variable. Statistical significance was set at $5 \%$.

\section{Results}

The mean age of the 4471 respondents was 38.3 years $(\mathrm{SD}=11.63)$ ranging from 18 to 85 years. More than half of the respondents were female (52.9\%), the majority had college/university education (80.9\%), did not lose their job during the pandemic $(91.9 \%)$, did not have reduced wages during the pandemic (73.2\%). Also, $15.7 \%$ had moderate risk of health problems and $91.3 \%$ reported not being depressed during the pandemic. There were 110 (2.5\%) respondents who tested positive for COVID-19. Table 1 highlights the demographic profiles of respondents.

There were 919 (20.5\%) respondents who reported living with HIV. PLWH were significantly older than respondents who were not living with HIV (40.43 vs 37.75 ; $\mathrm{p}<0.001$ ). Compared to respondents not living with HIV, a greater number of PLWH were female ( $\mathrm{p}=0.001)$, had no formal education or primary school education $(\mathrm{p}<0.001)$, were at high $(\mathrm{p}=0.008)$ or moderate $(\mathrm{p}=0.011)$ risk of health problems, either lost their employment $(\mathrm{p}=0.032)$ or had reduced wages $(p=0.002)$ during the pandemic, and felt depressed $(\mathrm{p}<0.001)$.

Significantly fewer PLWH tested positive for COVID$19(\mathrm{p}<0.001)$, had a close friend who tested positive for COVID-19 or knew someone who died from COVID-19 ( $p<0.001)$, kept physical distance $(\mathrm{p}<0.001)$, isolated/ quarantined $(\mathrm{p}<0.001)$, and worked remotely $(<0.001)$. Also, significantly more PLWH reported a decrease in food intake $(\mathrm{p}<0.001)$, feeling hungry but not eating $(\mathrm{p}<0.001)$ and cutting their size of meals or skipping meals $(\mathrm{p}<0.001)$.

The omnibus test of model coefficients for the logistic regression analysis highlighted in Table 2 indicates that the current models outperformed the null models. The respondents' HIV status was associated with adopting COVID-19 precautionary measures. PLWH had significantly lower odds of physical distancing when compared with people not living with HIV (AOR: 0.67). The odds of adopting physical distancing were also significantly lower for respondents who were older (AOR: 0.97); had primary, secondary and college/university education when compared with respondents with no formal education ( $\mathrm{p}<0.05)$; had lost a job (AOR: 0.70 ); had reduction in wages (AOR: 0.73 ); had a close friend who had tested positive for COVID-19 (AOR: 0.64); knew someone who had died from COVID-19 (AOR: 0.7); and had increased access to food (AOR: 0.71). Factors significantly associated with higher odds of adopting physical distancing were a high risk of health problems (AOR: 1.45); having COVID-19 symptoms but not taking a test (AOR: 1.35); and having no change in food access (AOR: 1.42).

PLWH had significantly lower odds of being isolated or quarantined (AOR: 0.62) when compared to people not living with HIV. Also, lower odds of isolating or quarantining were significantly associated with low risk of health problems (AOR: 0.67); testing positive for COVID-19 (AOR: 0.18); having symptoms but not testing for COVID-19 (AOR: 0.29); having a close friend who tested positive for COVID-19 (AOR: 0.40); knowing someone who died from COVID-19 (AOR: 0.60); and being depressed (AOR: 0.58). Older respondents had significantly higher odds of being isolated or quarantined (AOR: 1.01).

PLWH had significantly lower odds of working remotely (AOR: 0.37). Older respondents (AOR: 0.98); and respondents with college/university education (AOR: 0.30 ); reduced wages (AOR: 0.61); who felt depressed (AOR: 0.77) had significantly lower odds of working remotely. Respondents who lost a job (AOR: 1.61) and who reported no change in food access (AOR: 1.44) had significantly higher odds of working remotely.

The omnibus test of model coefficients for the logistic regression analysis highlighted in Table 3 indicates that the current models outperformed the null models. The Table presents the factors associated with food security indicators such as being hungry and not eating and having to cut the size of meals/skip meals. PLWH had significantly lower odds of being hungry and not eating compared to those not living with HIV (AOR: 0.24). Also, respondents who were younger (AOR: 0.98); had moderate risk of health problems (AOR: 0.77); had a close friend who tested positive for COVID-19 (AOR: 0.47); and who knew someone who died from COVID-19 (AOR: 0.76) had significantly lower odds of being hungry and not eating. Respondents who had lost a job (AOR: 14.06), had reduced wages (AOR: 5.70), had COVID-19 symptoms but were not tested $(\mathrm{AOR}=1.32)$ and who felt depressed (AOR: 1.34) had significantly higher odds of being hungry and not eating.

PLWH had significantly higher odds of cutting the size of meals or skipping meals during the pandemic than respondents not living with HIV (AOR: 3.18). Similarly, 
Table 1 Profile of respondents who reported their HIV status $(\mathrm{N}=4471)$

\begin{tabular}{|c|c|c|c|c|c|}
\hline Variables & $\begin{array}{l}\text { Total } \\
\mathrm{N}=4471 \\
\mathrm{n}(\%)\end{array}$ & $\begin{array}{l}\text { Not living with HIV } \\
\mathrm{N}=3552 \\
\mathrm{n}(\%)\end{array}$ & $\begin{array}{l}\text { Living with HIV } \\
\mathrm{N}=919 \\
\mathrm{n}(\%)\end{array}$ & Chi square/t test & p-value \\
\hline $\begin{array}{l}\text { Age } \\
\text { Mean (SD) in years }\end{array}$ & $38.30(11.63)$ & $37.75(11.76)$ & $40.43(10.84)$ & 15.25 & $<0.001$ \\
\hline \multicolumn{6}{|l|}{ Sex } \\
\hline Male & $2076(46.4)$ & $1705(48.0)$ & $371(40.4)$ & \multirow[t]{4}{*}{17.38} & \multirow[t]{4}{*}{0.001} \\
\hline Female & $2363(52.9)$ & $1822(52.1)$ & $541(58.9)$ & & \\
\hline Intersex & $3(0.1)$ & $2(0.1)$ & $1(0.1)$ & & \\
\hline Decline to answer & $29(0.6)$ & $23(0.6)$ & $6(0.7)$ & & \\
\hline \multicolumn{6}{|l|}{ Level of education } \\
\hline No Education & $48(1.1)$ & $7(0.2)$ & $41(4.5)$ & \multirow[t]{4}{*}{819.37} & \multirow[t]{4}{*}{$<0.001$} \\
\hline Primary & $84(1.9)$ & $7(0.2)$ & $77(8.4)$ & & \\
\hline Secondary & $724(16.2)$ & $386(10.9)$ & $338(36.8)$ & & \\
\hline College/university & 3615 (80.9) & $3152(88.7)$ & $463(50.4)$ & & \\
\hline \multicolumn{6}{|l|}{ Employment status } \\
\hline \multicolumn{6}{|l|}{ Job loss } \\
\hline No & $4110(91.9)$ & $3281(92.4)$ & $829(90.2)$ & \multirow[t]{2}{*}{$4.60 / 1$} & \multirow[t]{2}{*}{0.032} \\
\hline Yes & $361(8.1)$ & $271(7.6)$ & $90(9.8)$ & & \\
\hline \multicolumn{6}{|l|}{ Had reduced wages } \\
\hline No & $3271(73.2)$ & $2636(74.2)$ & $635(69.1)$ & \multirow[t]{2}{*}{9.73} & \multirow[t]{2}{*}{0.002} \\
\hline Yes & $1200(26.8)$ & $916(25.8)$ & $284(30.9)$ & & \\
\hline \multicolumn{6}{|l|}{ Health profile } \\
\hline \multicolumn{6}{|l|}{ High risk } \\
\hline No & $4304(96.3)$ & 3433 (96.6) & $871(94.8)$ & \multirow[t]{2}{*}{7.12} & \multirow[t]{2}{*}{0.008} \\
\hline Yes & $167(3.7)$ & $119(3.4)$ & $48(5.2)$ & & \\
\hline \multicolumn{6}{|l|}{ Moderate risk } \\
\hline No & $3770(84.3)$ & $3020(85.0)$ & $750(81.6)$ & \multirow[t]{2}{*}{6.43} & \multirow[t]{2}{*}{0.011} \\
\hline Yes & $701(15.7)$ & $532(15.0)$ & $169(18.4)$ & & \\
\hline \multicolumn{6}{|l|}{ Low risk } \\
\hline No & $4016(89.8)$ & $3197(90.0)$ & $819(89.1)$ & \multirow[t]{2}{*}{0.63} & \multirow[t]{2}{*}{0.428} \\
\hline Yes & 455 (10.2) & $355(10.0)$ & $100(10.9)$ & & \\
\hline COVID-19 status & & & & & \\
\hline Tested COVID-19 & & & & & \\
\hline No & $4361(97.5)$ & $3450(97.1)$ & $911(99.1)$ & 12.18 & $<0.001$ \\
\hline Yes & $110(2.5)$ & $102(2.9)$ & $8(0.9)$ & & \\
\hline Had symptoms but & & & & & \\
\hline No & 4004 (89.6) & $3178(89.5)$ & $826(89.5)$ & 0.13 & 0.717 \\
\hline Yes & 467 (10.4) & $374(10.5)$ & $93(10.1)$ & & \\
\hline Had a close friend & d positive for $\mathrm{C}$ & & & & \\
\hline No & 3753 (83.9) & 2917 (82.1) & $836(91.0)$ & 42.37 & $<0.001$ \\
\hline Yes & $718(16.1)$ & $635(17.9)$ & $83(9.0)$ & & \\
\hline Knew someone wh & m COVID-19 & & & & \\
\hline No & $3098(69.3)$ & $2353(66.2)$ & $745(81.1)$ & 75.38 & $<0.001$ \\
\hline Yes & $1373(30.7)$ & $1199(33.8)$ & $174(12.7)$ & & \\
\hline COVID-19 behavior & & & & & \\
\hline Physical distancing & & & & & \\
\hline No & $1239(27.7)$ & $895(25.2)$ & 344 (37.4) & 54.56 & $<0.001$ \\
\hline Yes & $3232(72.3)$ & $2657(74.8)$ & $575(62.6)$ & & \\
\hline Wearing mask or fa & & & & & \\
\hline
\end{tabular}


Table 1 (continued)

\begin{tabular}{|c|c|c|c|c|c|}
\hline Variables & $\begin{array}{l}\text { Total } \\
\mathrm{N}=4471 \\
\mathrm{n}(\%)\end{array}$ & $\begin{array}{l}\text { Not living with HIV } \\
\mathrm{N}=3552 \\
\mathrm{n}(\%)\end{array}$ & $\begin{array}{l}\text { Living with HIV } \\
\mathrm{N}=919 \\
\mathrm{n}(\%)\end{array}$ & Chi square/t test & p-value \\
\hline No & $941(21.0)$ & $759(21.4)$ & $182(19.8)$ & 1.08 & 0.300 \\
\hline Yes & $3530(79.0)$ & $2793(78.6)$ & $737(81.2)$ & & \\
\hline \multicolumn{6}{|c|}{ Washing or sanitizing hands more often } \\
\hline No & $1005(22.5)$ & $785(22.1)$ & $220(23.9)$ & 1.42 & 0.234 \\
\hline Yes & $3466(77.5)$ & $2767(77.9)$ & $699(76.1)$ & & \\
\hline \multicolumn{6}{|c|}{ Isolation/quarantine } \\
\hline No & $4128(92.3)$ & $3251(91.5)$ & $877(95.4)$ & 15.71 & $<0.001$ \\
\hline Yes & $343(7.7)$ & $301(8.5)$ & $42(4.6)$ & & \\
\hline \multicolumn{6}{|c|}{ Work remotely } \\
\hline No & $3098(69.3)$ & $2311(65.1)$ & 787 (85.6) & 145.24 & $<0.001$ \\
\hline Yes & $1373(30.7)$ & $1241(34.9)$ & $132(14.4)$ & & \\
\hline \multicolumn{6}{|l|}{ Food access } \\
\hline \multicolumn{6}{|l|}{ Food intake } \\
\hline Decreased & $913(22.0)$ & $629(19.1)$ & $284(32.7)$ & 103.16 & $<0.001$ \\
\hline Increased & $1511(36.3)$ & $1200(36.5)$ & $311(35.8)$ & & \\
\hline No change & 1734 (41.7) & $1461(44.5)$ & $273(31.4)$ & & \\
\hline \multicolumn{6}{|c|}{ Hungry but did not eat } \\
\hline No & $3183(71.2)$ & $2736(77.0)$ & 447 (48.6) & 286.87 & $<0.001$ \\
\hline Yes & $1288(28.8)$ & $816(23.0)$ & $472(51.4)$ & & \\
\hline \multicolumn{6}{|c|}{ Cut the size of meals or skip meals } \\
\hline No & $3942(65.8)$ & $2515(70.8)$ & $427(46.5)$ & 192.24 & $<0.001$ \\
\hline Yes & $1529(34.2)$ & $1037(29.2)$ & $492(53.5)$ & & \\
\hline \multicolumn{6}{|c|}{ Depressed during the pandemic } \\
\hline No & $4080(91.3)$ & $3299(92.9)$ & $781(85.0)$ & 57.00 & $<0.001$ \\
\hline Yes & $391(8.7)$ & $253(7.1)$ & $138(15.0)$ & & \\
\hline
\end{tabular}

male respondents (AOR: 1.19); and respondents who had lost their jobs (AOR: 16.07), who had had their wages reduced (AOR: 6.84), who adhered to physical distancing (AOR: 1.19) and who felt depressed (AOR: 1.39) had significantly higher odds of cutting the size of meals or skipping meals during the pandemic. Younger respondents (AOR: 0.98 ); and respondents with moderate risk of health problems (AOR: 0.77), had a close friend who tested positive to COVID-19 (AOR: 0.45) and who knew someone that died from COVID-19 (AOR: 0.84) had significantly lower odds of cutting the size of meals or skipping meals during the pandemic.

\section{Discussion}

This study findings indicates that PLWH were less likely to adopt COVID-19 prevention behaviors-physical distancing, isolation/quarantine and working remotely-when compared to people not living with HIV. Also, while both people living with and without HIV faced food security challenges during the COVID-19 pandemic, the responses differed between the two groups: people not living with HIV were more likely to go hungry without food while PLWH were more likely to cut food sizes and skip meals as a response strategy. The study hypothesis that PLWH are more likely to adopt COVID-19 risk preventive behaviors is not supported by the findings; and the hypothesis that PLWH are more likely to experience food security challenges when compared to people not living with HIV is partially supported.

One of the strengths of the study is the insightful analysis on the impact of the COVID-19 pandemic on the preventive behavior and food security of people living with and without HIV in Nigeria. It is one of the few studies on the impact of COVID-19 on PLWH in Africa; and an important contribution to the literature to help inform strategic actions for providing care and support for PLWH during the pandemic. The study however has a few limitations which suggest the need for cautious interpretation of the results. Firstly, the cross-sectional nature of the study makes it difficult to establish causality. In addition, the pandemic had multiple phases which may have had 
Table 2 Logistic regression analysis of factors associated with adopting COVID-19 precautionary measures (physical distancing, isolation/quarantine and working remotely) by adults in Nigeria $(\mathrm{N}=4471)$

\begin{tabular}{|c|c|c|c|c|c|c|}
\hline \multirow[t]{2}{*}{ Variables } & \multicolumn{2}{|c|}{ Physical distancing } & \multicolumn{2}{|l|}{ Isolation/quarantine } & \multicolumn{2}{|l|}{ Work remotely } \\
\hline & AOR $(95 \% \mathrm{CI})$ & $\mathrm{p}$ value & AOR $(95 \% \mathrm{CI})$ & $\mathrm{p}$ value & AOR $(95 \% \mathrm{CI})$ & $\mathrm{p}$ value \\
\hline Age in years & $0.97(0.97-0.98)$ & $<0.001$ & $1.01(1.00-1.03)$ & 0.039 & $0.98(0.98-0.99)$ & $<0.001$ \\
\hline \multicolumn{7}{|l|}{ Sex } \\
\hline Male (ref: Not male) & $0.94(0.82-1.08)$ & 0.412 & $0.92(0.72-1.17)$ & 0.481 & $0.90(0.79-1.03)$ & 0.128 \\
\hline \multicolumn{7}{|l|}{ HIV status } \\
\hline Living with HIV (ref: Not living with HIV) & $0.67(0.55-0.81)$ & $<0.001$ & $0.62(0.42-0.92)$ & 0.018 & $0.37(0.30-0.46)$ & $<0.001$ \\
\hline \multicolumn{7}{|l|}{ Level of education } \\
\hline No education & 1.00 & - & 1.00 & - & 1.00 & - \\
\hline Primary & $0.41(0.19-0.89)$ & 0.024 & $3.87(0.60-24.80)$ & 0.153 & $0.98(0.29-3.35)$ & 0.971 \\
\hline Secondary & $0.20(0.11-0.39)$ & $<0.001$ & $1.73(0.53-5.61)$ & 0.362 & $0.67(0.25-1.79)$ & 0.424 \\
\hline College/university & $0.14(0.07-0.26)$ & $<0.001$ & $1.39(0.44-4.38)$ & 0.577 & $0.30(0.11-0.78)$ & 0.014 \\
\hline \multicolumn{7}{|l|}{ Employment status } \\
\hline \multicolumn{7}{|l|}{ Job loss } \\
\hline Yes (ref: No) & $0.70(0.53-0.93)$ & 0.013 & $1.13(0.70-1.81)$ & 0.617 & $1.61(1.20-2.18)$ & 0.002 \\
\hline \multicolumn{7}{|l|}{ Had reduced wages } \\
\hline Yes (ref: No) & $0.73(0.61-0.87)$ & 0.001 & $0.86(0.65-1.14)$ & 0.290 & $0.61(0.52-0.71)$ & $<0.001$ \\
\hline \multicolumn{7}{|l|}{ Medical health profile } \\
\hline \multicolumn{7}{|l|}{ High risk } \\
\hline Yes (ref: No) & $1.45(1.00-2.09)$ & 0.050 & $1.17(0.63-2.19)$ & 0.622 & $0.94(0.66-1.34)$ & 0.742 \\
\hline \multicolumn{7}{|l|}{ Moderate risk } \\
\hline Yes (ref: No) & $1.08(0.88-1.33)$ & 0.460 & $0.83(0.60-1.14)$ & 0.252 & $1.01(0.83-1.23)$ & 0.905 \\
\hline \multicolumn{7}{|l|}{ Low risk } \\
\hline Yes (ref: No) & $0.82(0.64-1.05)$ & 0.114 & $0.67(0.48-0.94)$ & 0.020 & $0.89(0.71-1.11)$ & 0.315 \\
\hline \multicolumn{7}{|l|}{ COVID-19 status } \\
\hline \multicolumn{7}{|l|}{ Tested COVID-19 positive } \\
\hline Yes (ref: No) & $1.54(0.99-2.40)$ & 0.056 & $0.18(0.12-0.28)$ & $<0.001$ & $1.10(0.71-1.69)$ & 0.673 \\
\hline \multicolumn{7}{|l|}{ Had symptoms but did not test for COVID-19 } \\
\hline Yes (ref: No) & $1.35(1.08-1.69)$ & 0.009 & $0.29(0.23-0.40)$ & $<0.001$ & $1.04(0.83-1.31)$ & 0.707 \\
\hline \multicolumn{7}{|c|}{ Had a close friend who tested positive for COVID-19 } \\
\hline Yes (ref: No) & $0.64(0.51-0.79)$ & $<0.001$ & $0.40(0.31-0.53)$ & $<0.001$ & $1.02(0.84-1.23)$ & 0.866 \\
\hline \multicolumn{7}{|l|}{ Knew someone who died from COVID-19 } \\
\hline Yes (ref: No) & $0.71(0.60-0.83)$ & $<0.001$ & $0.60(0.47-0.78)$ & $<0.001$ & $0.93(0.80-1.08)$ & 0.335 \\
\hline \multicolumn{7}{|l|}{ Food access } \\
\hline \multicolumn{7}{|l|}{ Food intake } \\
\hline Decreased & 1.00 & - & 1.00 & - & 1.00 & - \\
\hline Increased & $0.71(0.58-0.86)$ & $<0.001$ & $1.19(0.86-1.63)$ & 0.294 & $0.87(0.72-1.05)$ & 0.136 \\
\hline No Change & $1.42(1.18-1.71)$ & $<0.001$ & $1.36(0.98-1.87)$ & 0.063 & $1.44(1.19-1.74)$ & $<0.001$ \\
\hline \multicolumn{7}{|l|}{ Hungry but did not eat } \\
\hline Yes (ref: No) & $1.07(0.81-1.41)$ & 0.640 & $1.28(0.82-2.02)$ & 0.282 & $0.83(0.65-1.07)$ & 0.153 \\
\hline \multicolumn{7}{|l|}{ Cut the size of meals or skip meals } \\
\hline Yes (ref: No) & $0.84(0.65-1.10)$ & 0.250 & $0.80(0.51-1.24)$ & 0.314 & $1.08(0.85-1.37)$ & 0.544 \\
\hline Depression & & & & & & \\
\hline Yes (ref: No) & $0.84(0.65-1.10)$ & 0.199 & $0.58(0.40-0.84)$ & 0.004 & $0.77(0.60-0.99)$ & 0.047 \\
\hline Nagelkerke $\mathrm{R}^{2}$ & 0.106 & $<0.001$ & 0.184 & $<0.001$ & 0.124 & $<0.001$ \\
\hline Omnibus test of model coefficients & 342.52 & $<0.001$ & 357.59 & $<0.001$ & 410.13 & $<0.001$ \\
\hline
\end{tabular}

$A O R$ adjusted odds ratio, $C I$ confidence interval 
Table 3 Logistic regression analysis of factors associated with food insecurity (hungry but did not eat, cut the size of meals or skip meal) during COVID-19 by adults in Nigeria $(\mathrm{N}=4471)$

\begin{tabular}{|c|c|c|c|c|}
\hline \multirow[t]{2}{*}{ Variables } & \multicolumn{2}{|l|}{ Hungry but did not eat } & \multicolumn{2}{|c|}{ Cut the size of meals or skip meals } \\
\hline & AOR $(95 \% \mathrm{CI})$ & $\mathrm{P}$ value & $\operatorname{AOR}(95 \% \mathrm{CI})$ & $P$ value \\
\hline Age in years & $0.98(0.97-0.99)$ & $<0.001$ & $0.98(0.98-0.99)$ & $<0.001$ \\
\hline \multicolumn{5}{|l|}{ Sex } \\
\hline Male (ref: Not male) & $1.15(0.98-1.34)$ & 0.083 & $1.19(1.02-1.38)$ & 0.023 \\
\hline \multicolumn{5}{|l|}{ HIV status } \\
\hline Living with HIV (ref: Not living with HIV) & $0.24(0.20-0.30)$ & $<0.001$ & $3.18(2.60-3.88)$ & $<0.001$ \\
\hline \multicolumn{5}{|l|}{ Level of education } \\
\hline No formal education & 1.00 & - & 1.00 & - \\
\hline Primary & $0.73(0.33-1.61)$ & 0.437 & $0.47(0.21-1.07)$ & 0.073 \\
\hline Secondary & $0.77(0.39-1.50)$ & 0.440 & $0.54(0.27-1.07)$ & 0.079 \\
\hline Tertiary & $0.75(0.38-1.45)$ & 0.394 & $0.58(0.29-1.13)$ & 0.109 \\
\hline \multicolumn{5}{|l|}{ Employment status } \\
\hline \multicolumn{5}{|l|}{ Job loss } \\
\hline Yes (ref: No) & $14.06(10.67-18.53)$ & $<0.001$ & $16.07(11.95-21.63)$ & $<0.001$ \\
\hline \multicolumn{5}{|l|}{ Had reduced wages } \\
\hline Yes (ref: No) & $5.70(4.84-6.72)$ & $<0.001$ & $6.84(5.83-8.01)$ & $<0.001$ \\
\hline \multicolumn{5}{|l|}{ Medical health profile } \\
\hline \multicolumn{5}{|l|}{ High risk } \\
\hline Yes (ref: No) & $1.18(0.77-1.80)$ & 0.456 & $1.05(0.70-1.58)$ & 0.807 \\
\hline \multicolumn{5}{|l|}{ Moderate risk } \\
\hline Yes (ref: No) & $0.77(0.61-0.97)$ & 0.025 & $0.77(0.61-0.95)$ & 0.017 \\
\hline \multicolumn{5}{|l|}{ Low risk } \\
\hline Yes (ref: No) & $1.15(0.89-1.48)$ & 0.275 & $1.12(0.88-1.43$ & 0.375 \\
\hline \multicolumn{5}{|l|}{ COVID-19 status } \\
\hline \multicolumn{5}{|l|}{ Tested COVID-19 positive } \\
\hline Yes (ref: No) & $1.12(0.65-1.95)$ & 0.682 & $1.07(0.63-1.82)$ & 0.795 \\
\hline \multicolumn{5}{|l|}{ Had symptoms but did not test for COVID-19 } \\
\hline Yes (ref: No) & $1.32(1.02-1.70)$ & 0.032 & $1.11(0.87-1.43)$ & 0.412 \\
\hline \multicolumn{5}{|c|}{ Had a close friend who tested positive for COVID-19 } \\
\hline Yes (ref: No) & $0.47(0.37-0.61)$ & $<0.001$ & $0.45(0.35-0.57)$ & $<0.001$ \\
\hline \multicolumn{5}{|l|}{ Knew someone who died from COVID-19 } \\
\hline Yes (ref: No) & $0.76(0.63-0.91)$ & 0.003 & $0.84(0.70-0.99)$ & 0.044 \\
\hline \multicolumn{5}{|l|}{ COVID-19 related behavioral changes } \\
\hline \multicolumn{5}{|l|}{ Physical distancing } \\
\hline Yes (ref: No) & $1.14(0.95-1.35)$ & 0.161 & $1.19(1.00-1.41)$ & 0.050 \\
\hline \multicolumn{5}{|l|}{ Isolation/self-quarantine } \\
\hline Yes (ref: No) & $1.11(0.82-1.50)$ & 0.514 & $0.97(0.72-1.31)$ & 0.839 \\
\hline \multicolumn{5}{|l|}{ Working from home } \\
\hline Yes (ref: No) & $0.91(0.77-1.09)$ & 0.312 & $0.99(0.84-1.17)$ & 0.901 \\
\hline \multicolumn{5}{|l|}{ Depression } \\
\hline Yes (ref: No) & $1.34(1.03-1.74)$ & 0.031 & $1.39(1.07-1.80)$ & 0.015 \\
\hline Nagelkerke $\mathrm{R}^{2}$ & 0.349 & $<0.001$ & 0.356 & $<0.001$ \\
\hline Omnibus test of model coefficients & 1250.16 & $<0.001$ & 1329.97 & $<0.001$ \\
\hline
\end{tabular}

$A O R$ adjusted odds ratio, $C I$ confidence interval

different effects on the population. Though the data was collected across these phases, the study could not capture the dynamics happening across the different phases due to the cross-sectional study design. Secondly, selfreport of depression is a more sensitive way of identifying non-depressed than depressed individuals [49] and this 
introduces potential underestimation of the proportion of respondents who are depressed. Thirdly, the sample was also skewed towards having higher levels of education, possibly a reflection of the web-based data collection strategy requiring respondents to have reliable access to the internet. The need for access to internet services may also have resulted in the exclusion of residents in rural Nigeria where internet infrastructure is poor. Fourthly, study participants were self- rather than randomly selected since the survey was distributed through social networks. However, we could only conduct a web-based survey during the pandemic because of the need for physical distancing recommended by health authorities [50]. Finally, the question asked about whether a close friend (and not about a family member) had contracted COVID-19 limited the ability of the study to assess the COVID-19 experience with family members since real-life interaction occur more often with family members than friends. Also, questions on COVID preventive measures adopted only had a 'yes' or 'no' response with no reflection on how often such precautions were used. Despite these limitations the study findings can still inform strategic decision-making.

First, the low level of adherence to COVID-19 prevention strategies among PLWH despite being at potentially greater risk for severe COVID-19 than people not living with HIV is a cause for concern. We noticed that a greater proportion of PLWH had no formal education or had primary school education increasing the possibility of them working in the large informal income sector in Nigeria [51]. The lockdown had a significant hard toll on people in the informal sector who survive on daily income [52]. The study result suggests that more PLWH lost their jobs and had reduced wages than people not living with HIV; and this implies PLWH may had had worse financial insecurity during the pandemic than people not living with HIV. This financial insecurity may be associated with inability to comply with COVID19 prevention strategies: physical distancing, isolation and quarantining will be challenging for individuals who live in crowded residential areas; the likely typology of residential areas people with poor financial security live [53]. Also, job opportunities available for the level of education of PLWH as portrayed in the present study reduce the prospect of working remotely.

PLWH are likely aware of their risk for contracting COVID-19 because of their immunocompromised status and associated risk of comorbidities [10]. Their inability to follow preventive measures despite their awareness of their risk suggests that PLWH may be facing more challenges than people not living with HIV. PLWH are at higher risk of depression than people not living with HIV [24] and food insecurity [20]; and the study showed that those who felt depressed were less likely to adopt COVID-19 prevention strategies and more likely to face food insecurity. This is further justifiable reason to increase support provided for PLWH during the pandemic.

We noticed that significantly fewer PLWH reported testing positive for COVID-19 in this study although there was no difference between both groups in reporting that they had the symptoms but did not take the test. This does not indicate that there are fewer PLWH who had contracted SARS-CoV-2 infection. Rather, this may be explained by lesser percentage of PLWH taking the COVID-19 test and suggesting the presence of structural barriers to COVID-19 testing for PLWH. In view of the higher risk of PLWH to SARS-CoV-2 infection and the higher risk of mortality [10], it is important to further study the reasons for the significantly lower percentage of positive COVID-19 test results reported for PLWH in this study. Where possible, PLWH should be prioritized for access to COVID-19 screening tests using structures through which they access their antiretroviral therapy.

Second, the study findings provide some insight into how PLWH manage their food security challenges: PLWH will rather cut down on size of the meals or number of rations and thereby save on food for another mealtime than go hungry. Although it may seem contradictory that PLWH had higher odds of reported cutting down on the size of the meals and number of rations than non-PLWH while the converse was the case for reporting on hunger, it is possible to reduce food portions without increasing hunger [54]. Skipping meals, however, has deleterious effects as its causes the metabolism to slow down with resultant weight gain over time [55]. PLWH may have adopted this food security measure as a strategy to ensure that antiretrovirals are not taken on an empty stomach. PLWH are often educated about the interactions between antiretrovirals and food and nutrition; and are educated about how to address nutritional issues to reduce their risk of poor food access. PLWH on antiretroviral therapy are prone to malnutrition due to inadequate appetite, dietary intake, nutritional losses, metabolic changes, and increased requirements for both macro- and micro-nutrients [56]. Poor food security may hasten progression to AIDS-related illnesses, undermine adherence and response to antiretroviral therapy which makes nutritional counselling an essential component of antiretroviral therapy [57]. Food security challenges for PLWH is a universal phenomenon [58] and thus, nutrition and food security counselling should be part of the routine therapy for PLWH. Having faced a life of food insecurity, PLWH may, therefore, be better prepared to manage food insecurity during this pandemic than people not living with HIV. Further studies are needed to validate the postulations generated from the study findings highlighted here.

Third, age was associated with COVID-19 related prevention behavior changes: older respondents were less likely to adopt physical distancing and to work remotely than younger 
respondents; while older respondents were more likely to adopt isolation/quarantine than younger respondents. Age is an independent risk factor for mortality in patients with COVID-19 being more fatal for older age groups because of their increased risk of comorbidities that can increase the risk of severe disease [59]. Age-dependent distancing measures focused on older population such as physical distancing and working remotely, could achieve a better balance between COVID-19 mortality and economic activity during the COVID-19 pandemic [60]. The study found that adoption of physical distancing measures (as well as working remotely) was more likely with younger than older adults in Nigeria. This finding indicates the need for strategic actions to improve adoption of COVID-19 preventive measures by older residents in Nigeria to balance COVID-19 mortality and economic activity during the pandemic.

Also, older respondents were less likely to be starve, cut the size of meals or skip meals. This finding may reflect the nature of African society where the care, including nutritional care, of older ones are prioritized. However, gender tends to moderate food security of persons in households in sub-Saharan Africa where males may be more food secure when families have fewer economic resources and females are more food secure when families have greater economic resources [61]. The only gender disparity observed in the current study was that male respondents were more likely to cut the size of meals or skip meals compared to non-male respondents during the pandemic. There is a need to further understand how Nigerians were protected from food insecurity during this pandemic.

Fourth, COVID-19 status was also associated with the adoption of COVID-19 preventive behaviors. Though we found that respondents who had symptoms but did not test for COVID-19 were more likely to adopt physical distancing, paradoxically, being tested positive to COVID-19 associated with lower odds of being isolated/quarantine. We posit that physical distancing may be perceived as a self-protecting practice likely to be adopted by those who suspect themselves of being infected in the hope that reducing additional risk of infection from others may avert disease progress. The decision to isolate or quarantine mainly protects others and may be more challenging to make for various reasons including stigma [62] and challenges with food access [63]. The study findings may also be a pointer to poor contact tracing and management in Nigeria [64]. Further studies are however needed to understand why people who had symptoms but did not test for COVID-19 were keep physical distancing as they may have had to struggle with structural barriers to COVID-19 testing [65].

Fifth, people who felt depressed were less likely to isolate/quarantine and more likely to face food insecurity than those who were not. Isolation is regarded as a public health infection control measure for persons who have contracted
COVID-19 while quarantine is for those in close contact with someone who may have had COVID-19 [66]. While there is information on COVID-19 being a risk factor for depression [67], there is little know about depression being a risk factor-mediator or moderator-for COVID-19. The study finding suggests that it is possible that people who are depressed who are made to isolate or quarantine may not adhere to this instruction. It may therefore be better to hospitalize persons with COVID-19 who feel depressed, irrespective of the severity of the COVID-19-to reduce the risk for spreading infection. Also, depression may reduce motivation to carry out daily activities, such as obtaining food or resources to get food thereby increasing the risk for food insecurity [68]. The study findings may suggest that persons who feel depressed during the pandemic need support to address food insecurity challenges, as well as compliances with isolation or quarantine directives.

Finally, some of the study findings were not unusual. People who lost their job and those who had a decrease in wage had significantly high risk for food insecurity. We also noticed what we identified unusual findings: respondents who had a close friend who tested positive for COVID-19 and those who knew someone who died from COVID-19 were less likely to have food security challenges; while respondents who had symptoms but did not test for COVID19 were more likely to be hungry and do not eat. We are unable to explain these unusual finding and suggest further studies to explore these findings considering the dynamic nature of the pandemic and the possible changes in the study outcomes as the pandemic unfolded.

\section{Conclusion}

In summary, the present study provides novel evidence that may explain the reported high risk of PLWH to a SARSCoV-2 infection: less PLWH than people without HIV adopt COVID-19 preventive behaviors. We also observed that older and depressed persons were less likely to adhere to COVID-19 preventive behaviors indicating the need for care and support of some sub-populations in the country. Also, PLWH were more likely than people not living with HIV to take food security measures that involved skipping a meal or cutting the size of food eaten rather than starve. The findings indicate that associations between adoption of COVID-19 preventive behaviors, mental and physical health, and food insecurity are highly complex; and in the light of some of the limitations acknowledged here, warrant further in-depth qualitative research that can unravel these incongruities.

Author Contributions MOF conceptualise the study and developed the first draft of the manuscript. OI conducted the statistical analysis. BB, 
MET, BU, OCE, NMA, GFA, EA, MAA, OOA, BEO, PE, BG, II, AOO, MJ, AT-AK, ZK, FBL, JL, NPN, BOP, MFAQ, MR, MRo, AS, ABA-T, MAY, RAAZ, JCO and ALN reviewed the multiple drafts of the manuscript and made intellectual inputs. All the authors agreed for the final version to be submitted for peer review and possible publication.

Funding Funding for the study was provided by the authors. Additionally, ALN was supported by the National Institutes of Health/National Institute on Aging (K01 AG064986).

Data Availability The data is accessible on request from the principal investigator.

Code Availability Not applicable.

\section{Declarations}

Conflict of interest The authors declare no conflict of interest.

Ethical Approval Ethical approval for the study was provided by the Institute of Public Health of the Obafemi Awolowo University Ile-Ife, Nigeria (HREC No: IPHOAU/12/1557).

Consent to Participate Study participants were required to tick a consent before proceeding to complete the survey after being duly informed about the study.

Consent for Publication Not applicable.

\section{References}

1. Gallo Marin B, Aghagoli G, Lavine K, et al. Predictors of COVID19 severity: a literature review. Rev Med Virol. 2021;31(1):1-10.

2. Zhou F, Yu T, Du R, et al. Clinical course and risk factors for mortality of adult inpatients with COVID-19 in Wuhan, China: a retrospective cohort study. Lancet. 2020;395:1054.

3. Soni SL, Kajal K, Yaddanapudi LN, et al. Demographic \& clinical profile of patients with COVID-19 at a tertiary care hospital in north India. Indian J Med Res. 2021;153(1 \& 2):115-25.

4. Mirzaei H, McFarland W, Karamouzian M, Sharifi H. COVID-19 among people living with HIV: a systematic review. AIDS Behav. 2021;25(1):85-92.

5. Vallée A, Fourn E, Majerholc C, Touche P, Zucman D. COVID-19 vaccine hesitancy among french people living with HIV. Vaccines. 2021;9(4):302.

6. Blanco JL, Ambrosioni J, Garcia F, et al. COVID-19 in patients with HIV: clinical case series. Lancet HIV. 2020;7(5):e314-6.

7. Del Amo J, Polo R, Moreno S, et al. Incidence and severity of COVID-19 in HIV-positive persons receiving antiretroviral therapy: a cohort study. Ann Intern Med. 2020;173:M20-3689.

8. Karmen-Tuohy S, Carlucci PM, Zacharioudakis IM, et al. Outcomes among HIVpositive patients hospitalized with COVID-19. J Acquir Immune Defic Syndr. 2020;85(1):6-10.

9. Gervasoni C, Meraviglia P, Riva A, et al. Clinical features and outcomes of HIV patients with coronavirus disease 2019. Clin Infect Dis. 2020. https://doi.org/10.1093/cid/ciaa579.

10. Ssentongo P, Heilbrunn ES, Ssentongo AE, et al. Epidemiology and outcomes of COVID-19 in HIV-infected individuals: a systematic review and meta-analysis. Sci Rep. 2021;11:6283.
11. Huang J, Xie N, Hu X, et al. Epidemiological, virological and serological features of coronavirus disease 2019 (COVID-19) cases in people living with human immunodeficiency virus in Wuhan: a population-based cohort study. Clin Infect Dis. 2020. https://doi.org/10.1093/cid/ciaa1186.

12. Jiang H, Zhou Y, Tang W. Maintaining HIV care during the COVID-19 pandemic. Lancet HIV. 2020;7(5):e308-9.

13. Waterfield KC, Shah GH, Etheredge GD, et al. Consequences of COVID-19 crisis for persons with HIV: the impact of social determinants of health. BMC Public Health. 2021;21:299.

14. Eghtessadi R, Mukandavire Z, Mutenherwa F, Cuadros D, Musuka G. Safeguarding gains in the sexual and reproductive health and AIDS response amidst COVID-19: the role of African civil society. Int J Infect Dis. 2020;100:286-91.

15. Mishra K, Rampal J. The COVID-19 pandemic and food insecurity: a viewpoint on India. World Dev. 2020;135:105068.

16. Core indicators of nutritional state for difficult-to-sample populations. J Nutr. 1990; 120 Suppl 11:1559-1600

17. The Lancet HIV. The syndemic threat of food insecurity and HIV. Lancet HIV. 2020;7(2):75.

18. Shiau S, Krause KD, Valera P, Swaminathan S, Halkitis PN. The burden of COVID-19 in people living with HIV: a syndemic perspective. AIDS Behav. 2020;4(8):2244-9.

19. Gundersen C, Ziliak JP. Food insecurity and health outcomes. Health Aff (Millwood). 2015;34(11):1830-9.

20. Jones AD. Food insecurity and mental health status: a global analysis of 149 countries. Am J Prev Med. 2017;53(2):264-73.

21. Santos GM, Ackerman B, Rao A, et al. Economic, mental health, HIV prevention and HIV treatment IMPACTS of COVID-19 and the COVID-19 response on a global sample of cisgender gay men and other men who have sex with men. AIDS Behav. 2021;25(2):311-21.

22. Tsai AC, Bangsberg DR, Frongillo EA, et al. Food insecurity, depression and the modifying role of social support among people living with HIV/AIDS in rural Uganda. Soc Sci Med. 2012;74(12):2012-9.

23. Anema A, Weiser SD, Fernandes KA, et al. High prevalence of food insecurity among HIV-infected individuals receiving HAART in a resource-rich setting. AIDS Care. 2011;23(2):221-30.

24. Vogenthaler NS, Hadley C, Rodriguez AE, Valverde EE, del Rio C, Metsch LR. Depressive symptoms and food insufficiency among HIV-infected crack users in Atlanta and Miami. AIDS Behav. 2011;15(7):1520152-6.

25. Seid S, Abdu O, Mitiku M, Tamirat KS. Prevalence of depression and associated factors among HIV/AIDS patients attending antiretroviral therapy clinic at Dessie referral hospital, South Wollo, Ethiopia. Int J Ment Health Syst. 2020;14:55.

26. Verger P, Lions C, Ventelou B. Is depression associated with health risk-related behaviour clusters in adults? Eur J Public Health. 2009;19(6):618-24.

27. Pike IL, Patil CL. Understanding women's burdens: preliminary findings on psychosocial health among Datoga and Iraqw women of northern Tanzania. Cult Med Psychiatry. 2006;30(3):299-330.

28. Ibukun CO, Adebayo AA. Household food security and covid-19 pandemic in Nigeria. https://www.uneca.org/sites/default/files/ AEC/2020/presentations/household_food_security_and_covid_ 19_ibukun_and_adebayo.pdf. Accessed 26 Apr 2021.

29. Awofala AA, Ogundele OE. HIV epidemiology in Nigeria. Saudi J Biol Sci. 2018;25(4):697-703.

30. UNAIDS. Press Release: New survey results indicate that Nigeria has an HIV prevalence of 1.4\%. 19 March 2019. https://www. unaids.org/en/resources/presscentre/pressreleaseandstatementa rchive/2019/march/20190314_nigeria. Accessed 28 Apr 2021.

31. Adepoju P. Nigeria's widening tuberculosis gap. Lancet Infect Dis. 2020;20(1):29. 
32. Musa BM, Musa B, Muhammed H, Ibrahim N, Musa AG. Incidence of tuberculosis and immunological profile of TB/HIV coinfected patients in Nigeria. Ann Thorac Med. 2015;10(3):185-92.

33. Schouten J, Wit FW, Stolte IG, et al. Cross-sectional comparison of the prevalence of age-associated comorbidities and their risk factors between HIV-infected and uninfected individuals: the AGEhIV cohort study. Clin Infect Dis. 2014;59(12):1787-97.

34. Anjorin AA, Abioye AI, Asowata OE, et al. Comorbidities and the COVID-19 pandemic dynamics in Africa. Trop Med Int Health. 2021;26(1):2-13.

35. Boulle A, Davies MA, Hussey H, et al. Risk factors for COVID19 death in a population cohort study from the Western Cape Province South Africa. Clin Infect Dis. 2020. https://doi.org/10. 1093/cid/ciaa1198.

36. Kalu B. COVID-19 in Nigeria: a disease of hunger. Lancet Respir Med. 2020;8(6):556-7.

37. Sholeye OO, Animasahun VJ, Salako AA, Oyewole BK. Household food insecurity among people living with HIV in Sagamu, Nigeria: a preliminary study. Nutr Health. 2017;23(2):95-102.

38. Pearlin LI. The sociological study of stress. J Health Soc Behav. 1989;30(3):241-56.

39. Pearlin LI, Lieberman MA, Menaghan EG, Mullan JT. The stress process. J Health Soc Behav. 1981;22(4):337-56.

40. Kessler RC, Price RH, Wortman CB. Social factors in psychopathology: stress, social support, and coping processes. Annu Rev Pschol. 1985;36:531-72.

41. Nguyen A, Brown B, El Tantawi M, Ndembi N, Okeibunor J, Mohammed A, Folayan M. Time to scale-up research collaborations to address the global impact of COVID-19-a commentary. Health Behav Policy Rev. 2021;8(3):277-80.

42. Nguyen AL, Christensen C, Taylor J, Brown B. Leaning on community-based participatory research to respond during COVID19. AIDS Behav. 2020;24(10):2773-5.

43. Marg LZ, Heidari O, Taylor J, et al. A multidimensional assessment of successful aging among older people living with HIV in palm springs, California. AIDS Res Hum Retroviruses. 2019;35(11-12):1174-80.

44. Centers for Disease Prevention and Control. People with Certain Medical Conditions. 29 April 2021. https://www.cdc.gov/coron avirus/2019-ncov/need-extra-precautions/people-with-medic al-conditions.html\#: :text=Chronic\%20lung\%20diseases\%2C\% 20including $\% 20$ COPD, severely $\% 20$ ill $\% 20$ from $\% 20$ COVID $\%$ 2D19. Accessed 1 May 2021.

45. Harkness A. The pandemic stress index. Florida: University of Miami; 2020.

46. U.S. household food security survey module: three-stage design, with screeners Economic Research Service, USDA September 2012. https://www.ers.usda.gov/media/8271/hh2012.pdf. Accessed 31 Oct 2020.

47. Van Selm M, Jankowski NW. Conducting online surveys. Qual Quant. 2006;4:435-56.

48. King DB, O'Rourke N, DeLongis A. Social media recruitment and online data collection: a beginner's guide and best practices for accessing low-prevalence and hard-to-reach populations. Can Psychol. 2014;55:240-9.

49. Zarghami M, Taghizadeh F, Moosazadeh M, Kheradmand M, Heydari K. Validity of self-reporting depression in the Tabari cohort study population. Neuropsychopharmacol Rep. 2020;40(4):342-7.

50. Hlatshwako TG, Shah SJ, Kosana P, et al. Online health survey research during COVID-19. Lancet Digit Health. 2021;3(2):e76-7.

51. Onwe OJ. Role of the informal sector in development of the Nigerian economy: output and employment approach. J Econ Dev Stud. 2013;1:60-74.
52. Duerksen, M. Innovations needed to prevent COVID-19 from catching fire in African cities. Africa Center for Strategic Studies. 2020. https://africacenter.org/spotlight/innovations-needed-preve nt-covid-19-catching-fire-africa-cities/. Accessed 30 Apr 2021.

53. Motoc I, Timmermans EJ, Deeg D, Penninx BWJH, Huisman M. Associations of neighbourhood sociodemographic characteristics with depressive and anxiety symptoms in older age: results from a 5-wave study over 15 years. Health Place. 2019;59:102172.

54. Jennings K-A. 8 Tips to Reduce Food Portions Without Increasing Hunger. Healthline. March 20, 2017. https://www.healthline.com/ nutrition/8-tips-to-reduce-portions. Accessed 18 July 2021.

55. Zeballos E, Todd JE. The effects of skipping a meal on daily energy intake and diet quality. Public Health Nutr. 2020;23(18):3346-55.

56. Waterfield KC, Shah GH, Etheredge GD, Ikhile O. Consequences of COVID-19 crisis for persons with HIV: the impact of social determinants of health. BMC Public Health. 2021;21(1):299.

57. ENN. Antiretroviral therapy and Nutrition: technical guidance. Field Exchange 20. 2003. https://www.ennonline.net/fex/20/thera py. Accessed 26 Apr 2021.

58. Weiser SD, Young SL, Cohen CR, Kushel MB, Tsai AC, Tien PC, et al. Conceptual framework for understanding the bidirectional links between food insecurity and HIV/AIDS. Am J Clin Nutr. 2011;94(6):1729S-1739S.

59. Sinclair AJ, Abdelhafiz AH. Age, frailty and diabetes-triple jeopardy for vulnerability to COVID-19 infection. EClinicalMedicine. 2020;22:100343.

60. Ortega-Quijano D, Ortega-Quijano N. Impact of age-selective vs non-selective physical-distancing measures against coronavirus disease 2019: a mathematical modelling study. Int J Epidemiol. 2021. https://doi.org/10.1093/ije/dyab043.

61. Masa R, Khan Z, Chowa G. Youth food insecurity in Ghana and South Africa: Prevalence, socioeconomic correlates, and moderation effect of gender. Child Youth Serv Rev. 2020;116:105180.

62. Ogunleye OO, Basu D, Mueller D, et al. Response to the novel corona virus (COVID-19) pandemic across Africa: successes, challenges, and implications for the future. Front Pharmacol. 2020;11:1205.

63. Chu IY, Alam P, Larson HJ, Lin L. Social consequences of mass quarantine during epidemics: a systematic review with implications for the COVID-19 response. J Travel Med. 2020;27(7):taaa192.

64. Nachega JB, Atteh R, Ihekweazu C, et al. Contact tracing and the COVID-19 response in Africa: best practices, key challenges, and lessons learned from Nigeria, Rwanda, South Africa, and Uganda. Am J Trop Med Hyg. 2021;104(4):1179-87.

65. Mills E, Nachega JB, Buchan I, Orbinski J, Attaran A, Singh S. Adherence to antiretroviral therapy in sub-Saharan Africa and North America. JAMA. 2006;296(6):679-90.

66. Narayana Health. COVID-19 Protocol: Isolation, Quarantine, Social Distancing [Internet]. 2020 [cited 2021 Jun 11]. Available from: https://www.narayanahealth.org/blog/covid-19-protocolisolation-quarantine-social-distancing/. Accessed 11 Aug 2021

67. Bueno-Notivol J, Gracia-García P, Olaya B, et al. Prevalence of depression during the COVID-19 outbreak: a meta-analysis of community-based studies. Int J Clin Health Psychol. 2021;21(1):100196.

68. Davey-Rothwell MA, Flamm LJ, Kassa HT, Latkin CA. Food insecurity and depressive symptoms: comparison of drug using and nondrug-using women at risk for HIV. J Community Psychol. 2014;42(4):469-78.

Publisher's Note Springer Nature remains neutral with regard to jurisdictional claims in published maps and institutional affiliations. 


\section{Authors and Affiliations}

Morenike Oluwatoyin Folayan ${ }^{1,2}(-)$. Olanrewaju Ibigbami ${ }^{3} \cdot$ Brandon Brown $^{1,4} \cdot$ Maha El Tantawi $^{1,5}$. Benjamin Uzochukwu ${ }^{1,6}$. Oliver C. Ezechi ${ }^{1,7}$. Nourhan M. Aly ${ }^{1,5}$. Giuliana Florencia Abeldaño ${ }^{1,8}$. Eshrat Ara ${ }^{1,9}$. Martin Amogre Ayanore ${ }^{1,10}$. Oluwagbemiga O. Ayoola ${ }^{1,11}$ - Bamidele Emmanuel Osamika ${ }^{1,12}$. Passent Ellakany ${ }^{1,13}$. Balgis Gaffar ${ }^{1,14}$. Ifeoma Idigbe ${ }^{1,7}$. Anthonia Omotola Ishabiyi ${ }^{1,15}$. Mohammed Jafer ${ }^{1,16}$. Abeedha Tu-Allah Khan ${ }^{1,17}$. Zumama Khalid ${ }^{1,18}$. Folake Barakat Lawal ${ }^{1,19}$. Joanne Lusher ${ }^{1,20}$. Ntombifuthi P. Nzimande ${ }^{1,21}$. Bamidele Olubukola Popoola ${ }^{1,22}$. Mir Faeq Ali Quadri ${ }^{1,23}$. Maher Rashwan ${ }^{1,24,25}$. Mark Roque $^{1,26}$. Anas Shamala ${ }^{1,27}$. Ala'a B. Al-Tammemi ${ }^{1,28}$. Muhammad Abrar Yousaf ${ }^{1,29}$. Roberto Ariel Abeldaño Zuñiga ${ }^{1,30}$. Joseph Chukwudi Okeibunor ${ }^{1,31}$ • Annie Lu Nguyen ${ }^{1,32}$

1 Mental Health and Wellness Study Group, Ile-Ife, Nigeria

2 Department of Child Dental Health, Obafemi Awolowo University, Ile-Ife, Nigeria

3 Department of Mental Health, Obafemi Awolowo University, Ile-Ife, Nigeria

4 Department of Social Medicine, Population and Public Health, Center for Healthy Communities, UCR School of Medicine, Riverside, CA, USA

5 Department of Pediatric Dentistry and Dental Public Health, Faculty of Dentistry, Alexandria University, Alexandria, Egypt

6 Department of Community Medicine, University of Nigeria Nsukka (Enugu Campus), Nsukka, Nigeria

7 Department of Clinical Sciences, Nigerian Institute of Medical Research, Lagos, Nigeria

8 School of Medicine, University of Sierra Sur, Oaxaca, Mexico

9 Department of Psychology, Government College for Women, Moulana Azad Road, Srinagar, Kashmir (J\&K) 190001, India

10 Department of Health Policy Planning and Management, University of Health and Allied Sciences, Ho, Ghana

11 Department of Radiology, Obafemi Awolowo University, Ile-Ife, Nigeria

12 Department of Psychology, Lead City University, Ibadan, Nigeria

13 Department of Substitutive Dental Sciences, College of Dentistry, Imam Abdulrahman Bin Faisal University, Dammam, Saudi Arabia

14 Department of Preventive Dental Sciences, College of Dentistry, Imam Abdulrahman Bin Faisal University, Dammam, Kingdom of Saudi Arabia

15 Centre for Rural Health, School of Nursing and Public Health, University of KwaZulu-Natal, Durban, South Africa

16 Department of Health Promotion, Faculty of Health, Medicine, and Life Sciences, Maastricht University, Maastricht, The Netherlands
17 School of Biological Sciences, University of the Punjab, Quaid-i-Azam Campus, Lahore 54590, Pakistan

18 School of Biological Sciences, University of the Punjab, Lahore 54590, Pakistan

19 Department of Periodontology and Community Dentistry, College of Medicine, University of Ibadan, Ibadan, Nigeria

20 School of Health and Life Sciences, University of the West of Scotland, London, UK

21 Department of Economic and Human Geography, University of Szeged, Szeged 6722, Hungary

22 Department of Child Oral Health, University of Ibadan, Ibadan, Nigeria

23 Department of Preventive Dental Sciences, Jazan University, Jizan, Saudi Arabia

24 Centre for Oral Bioengineering, Barts and the London, School of Medicine and Dentistry, Queen Mary University of London, Mile End Road, London E1 4NS, UK

25 Department of Conservative Dentistry, Faculty of Dentistry, Alexandria University, Alexandria, Egypt

26 Maternity \& Childhood Department, College of Nursing, Taibah University, Madinah 42356, Kingdom of Saudi Arabia

27 Department of Preventive and Biomedical Science, College of Dentistry, University of Science \& Technology, Sanaa, Yemen

28 Department of Family and Occupational Medicine, Faculty of Medicine, Doctoral School of Health Sciences, University of Debrecen, Debrecen, Hungary

29 Institute of Zoology, University of the Punjab, Quaid-i-Azam Campus, Lahore 54590, Pakistan

30 Postgraduate Department, University of Sierra Sur, Oaxaca, Mexico

31 World Health Organisation, AFRO, Addis Ababa, Ethiopia

32 Department of Family Medicine, Keck School of Medicine, University of Southern California, Los Angeles, CA, USA 